Published in final edited form as:

Synapse. 2017 August ; 71(8): . doi:10.1002/syn.21981.

\title{
$\left[{ }^{18} \mathrm{~F}\right]$ Nifene test-retest reproducibility in first-in-human imaging of $a 4 \beta 2^{*}$ nicotinic acetylcholine receptors
}

\author{
Patrick J. Lao ${ }^{1,2}$, Tobey J. Betthauser ${ }^{1,2}$, Dana L. Tudorascu ${ }^{3}$, Todd E. Barnhart ${ }^{1}$, Ansel T. \\ Hillmer $^{4}$, Charles K. Stone ${ }^{5}$, Jogeshwar Mukherjee ${ }^{6}$, and Bradley T. Christian ${ }^{1,2,7}$ \\ ${ }^{1}$ Department of Medical Physics, University of Wisconsin-Madison, Madison, Wisconsin 53705 \\ ${ }^{2}$ Waisman Laboratory for Brain Imaging and Behavior, Madison, Wisconsin 53705 \\ ${ }^{3}$ Department of Medicine, Biostatistics, Psychiatry, and Clinical and Translational Science, \\ University of Pittsburgh, Pittsburgh, Pennsylvania 15213 \\ ${ }^{4}$ Department of Radiology and Biomedical Imaging, and Psychiatry, Yale University, New Haven, \\ Connecticut 06520 \\ ${ }^{5}$ Department of Medicine, University of Wisconsin School of Medicine and Public Health, \\ Madison, Wisconsin 53705 \\ ${ }^{6}$ Department of Radiological Sciences, University of California-Irvine, Irvine, California 92697 \\ ${ }^{7}$ Department of Psychiatry, University of Wisconsin-Madison, Madison, Wisconsin 53705
}

\begin{abstract}
The aim of this study was to examine the suitability of $\left[{ }^{18} \mathrm{~F}\right]$ nifene, a novel a $4 \beta 2 *$ nicotinic acetylcholine receptor (nAChR) radiotracer, for in vivo brain imaging in a first-in-human study.

Methods-Eight healthy subjects (4 M,4 F;21-69,44 \pm 21 yrs) underwent a $\left[{ }^{18} \mathrm{~F}\right]$ nifene positron emission tomography scan $(200 \pm 3.7 \mathrm{MBq})$, and seven underwent a second scan within $58 \pm 31$ days. Regional estimates of DVR were measured using the multilinear reference tissue model (MRTM2) with the corpus callosum as reference region. DVR reproducibility was evaluated with test-retest variability (TRV) and intraclass correlation coefficient (ICC).
\end{abstract}

Results-The DVR ranged from 1.3 to 2.5 across brain regions with a TRV of $0-7 \%$, and did not demonstrate a systematic difference between test and retest. The ICCs ranged from 0.2 to 0.9 . DVR estimates were stable after $40 \mathrm{~min}$.

Conclusion-The binding profile and tracer kinetics of $\left[{ }^{18} \mathrm{~F}\right]$ nifene make it a promising a $4 \beta 2 *$ $\mathrm{nAChR}$ radiotracer for scientific research in humans, with reliable DVR test-retest reproducibility.

\section{Keywords}

a4ß2; first-in-humans; positron emission tomography

Correspondence: Patrick J. Lao, M.S., Department of Medical Physics, University of Wisconsin-Madison, 1500 Highland Ave., Madison,WI 53705.plao@wisc.edu.

CONFLICT OF INTEREST

The authors have no conflicts of interest to declare. 


\section{1 | INTRODUCTION}

Neuronal-type nicotinic acetylcholine receptors (nAChRs) are made of five subunits in homomeric or heteromeric combinations of $\alpha 2-\alpha 10$ and $\beta 2-\beta 4$ subunits (Gotti \& Clementi, 2004). Heteromeric combinations of the $a 4$ or $\beta 2$ subunits (e.g., $\alpha 4_{3} \beta 2_{2}$ or $a 4_{2} \beta 2_{3}$ ) represent the most abundant neuronal-type $\mathrm{nAChR}$ in the mammalian brain(Lind-strom et al., 1995), and any combination of the two are collectively referred to as a $4 \beta 2 *$ nAChRs. The $a 4 \beta 2 * n A C h R$ is a ligand-gated ion channel that is nonselectively permeable to $\mathrm{Na}^{+}$and $\mathrm{K}^{+}$, which can cause membrane depolarization leading to presynaptic and postsynap-tic excitation. The a $4 \beta 2 *$ nAChR system is involved in memory, attention, and mood, as well as the regulation of other neurotransmitters (Gotti \& Clementi, 2004). The expression of a $4 \beta 2 *$ nAChRs could provide clinically useful information about abnormalities in neurodevelop-ment (e.g., changes due to fetal alcohol exposure), substance abuse (e.g., tobacco and alcohol addiction), and neurological disease and disorders (e.g., Alzheimer's disease, Parkinson's disease, epilepsy, or schizophrenia), as well as healthy aging (Gotti \& Clementi, 2004; Hillmer et al., 2014).

The clinical and scientific relevance of $\alpha 4 \beta 2 *$ nAChRs demonstrates a need for a positron emission tomography (PET) radioligand for these receptors, particularly an agonist, which may be more sensitive to competing endogenous ligands than an antagonist. The first a $4 \beta 2 *$ $\mathrm{nAChR}$ agonist radioligand was $\left[{ }^{11} \mathrm{C}\right]$ nicotine; however, the high non-specific binding and rapid dissociation precluded its use in PET studies (Nybäck, Halldin, Åhlin, Curvall, \& Eriksson, 1994). A high-affinity radio-ligand, 2- $\left[{ }^{18} \mathrm{~F}\right] \mathrm{FA}-85380\left(\left[{ }^{18} \mathrm{~F}\right] 2 \mathrm{FA}\right)$, has been used extensively to investigate a $4 \beta 2 * \mathrm{nAChRs}$ in vivo in humans, but requires a long scan duration (approximately 6-8 hr) for stable parameter estimation following bolus injection or bolus plus infusion administration (Kimes et al., 2008). The azetidine ring responsible for the high affinity of $\left[{ }^{18} \mathrm{~F}\right] 2 \mathrm{FA}$ also results in slower in vivo kinetics. Therefore, the azetidine ring was replaced by a 3-pyrroline ring while preserving the pyridine ring to maintain the agonist properties of $\left[{ }^{18} \mathrm{~F}\right] 2 \mathrm{FA}$ (Constantinescu, Garcia, Mirbolooki, Pan, \& Mukherjee, 2013; Pichika, Easwaramoorthy, Collins, \& Mukherjee, 2006). The resulting compound, 2$\left[{ }^{18} \mathrm{~F}\right]$-fluoro-3-[2-((S)-3-pyrrolinyl)methoxy]pyridine (abbreviated: $\left[{ }^{18} \mathrm{~F}\right]$ nifene), is a moderate affinity $\left(K_{\mathrm{i}}=0.50 \mathrm{nM}\right)$ agonist radioligand for $\mathrm{a} 4 \beta 2 * \mathrm{nAChRs}$, which is approximately three times that of nicotine in rat brain homogenates $\left(K_{\mathrm{i}}=1.68 \mathrm{nM}\right)($ Pichika et al., 2006). Nifene exhibited subnanomolar affinities for the cloned human a $2 \beta 2(0.34$ $\mathrm{nM}), \alpha 3 \beta 2(0.80 \mathrm{nM})$, and $\mathrm{a} 4 \beta 2(0.83 \mathrm{nM})$ subtypes in rats and was found to be significantly weaker for other human nAChR subtypes (Liang, Patel, Pan, \& Mukher-jee, 2016). $\left[{ }^{18} \mathrm{~F}\right]$ Nifene demonstrated rapid and reversible kinetics, as well as a binding profile similar to the known brain distribution of $a 4 \beta 2 * \mathrm{nAChRs}$ in mice, rats, and non-human primates (Constantinescu et al., 2013; Hillmer, Wooten, Farhoud, et al., 2013; Hillmer et al., 2012; Pichika et al., 2006). More recently, several $\left[{ }^{18} \mathrm{~F}\right]$ radiotracers with faster kinetic properties than $\left[{ }^{18} \mathrm{~F}\right] 2 \mathrm{FA}$ have been developed and investigated in nonhuman primates. $\left[{ }^{18} \mathrm{~F}\right]$ Nifene had faster kinetics than $\left[{ }^{18} \mathrm{~F}\right] \mathrm{AZAN},\left[{ }^{18} \mathrm{~F}\right] \mathrm{XTRA}$, and $\left[{ }^{18} \mathrm{~F}\right]$ flubatine, but with lower $\mathrm{BP}_{\mathrm{ND}}$ values in $\alpha 4 \beta 2 * \mathrm{nAChR}$ rich regions in preclinical evaluation (Kuwabara et al., 2016). 
Estimated radiation burden of a $\left[{ }^{18} \mathrm{~F}\right]$ nifene PET scan based upon mouse dosimetry studies was similar in magnitude to that of a $\left[{ }^{18} \mathrm{~F}\right] 2 \mathrm{FA}$ PET scan, with $\left[{ }^{18} \mathrm{~F}\right]$ nifene having a relatively smaller dose and allows for multiple injections per year (Constantinescu et al., 2013). Dynamic $\left[{ }^{18} \mathrm{~F}\right]$ nifene data can be described by a 1-tissue compartment model with an arterial input function in nonhuman primates (Hillmer et al., 2012). Reference regions approaches using the cerebellum as reference demonstrated close agreement of estimated binding parameters $\left(\mathrm{BP}_{\mathrm{ND}}\right.$, DVR) with those of the 1-tissue compartmental model with arterial sampling (Hillmer et al., 2012). However, in humans, the cerebellum demonstrates non-negligible specific $\left[{ }^{18} \mathrm{~F}\right] 2 \mathrm{FA}$ binding, while the corpus callosum could serve as a more suitable reference region (Brody et al., 2006). Reference region methods are widely used to estimate neuroreceptor binding and are often preferred since they eliminate the need for invasive and complex measurement of metabolite-corrected arterial input functions.

The goal of this work was to perform a first-in-human $\left[{ }^{18} \mathrm{~F}\right]$ nifene PET study in which we evaluated the range and distribution of DVR values in the healthy brain, test-retest reproducibility, and minimum scan duration.

\section{2 | METHODS}

\section{1 | Subjects}

Eight healthy subjects ( $44 \pm 21$ years; $4 \mathrm{M}, 4 \mathrm{~F}$ ) participated after providing written informed consent. Inclusion criteria were 18- to 88-year old and good health. Exclusion criteria included any psychotropic medication that interacts with $a 4 \beta 2 *$ nAChRs or a positive pregnancy test. Procedures were conducted under the approval of the Institutional Review Board at the University of Wisconsin-Madison. As part of this first-in-human study, subjects also underwent a physical exam, blood chemistry profile, complete blood count, and urinalysis before and after radiotracer administration at test and retest.

\section{2 | PET acquisition}

$\left[{ }^{18} \mathrm{~F}\right]$ Nifene was synthesized in-house using previously published methods (Hillmer et al., 2011 ) with specific activities of $718 \pm 359 \mathrm{MBq} / \mathrm{nmol}$ and injected doses of $200 \pm 3.7 \mathrm{MBq}$ (20-30 s bolus via the ante-cubital vein). The administered mass of [ $\left.{ }^{18} \mathrm{~F}\right]$ nifene was $.07 \pm .04$ $\mu \mathrm{g}(.04-0.16 \mu \mathrm{g})$ and $.06 \pm .04 \mu \mathrm{g}(.03-0.12 \mu \mathrm{g})$ for test and retest, respectively. After a 6-min transmission scan $\left({ }^{68} \mathrm{Ge} /{ }^{68} \mathrm{Ga}\right.$ rotating rod sources), eight subjects underwent dynamic $\left[{ }^{18} \mathrm{~F}\right]$ nifene PET scan on a ECAT EXACT HR+ PET scanner (Siemens) in 3 D mode for 90 $\min (8 \times 0.5 \mathrm{~min}, 3 \times 2 \mathrm{~min}, 16 \times 5 \mathrm{~min}$ frames $)$. Seven subjects $(44 \pm 21$ years;3 M,4 F) underwent a second $\left[{ }^{18} \mathrm{~F}\right]$ nifene PET scan $58 \pm 31$ days later. There was no significant difference in the injected mass of nifene between the test and retest scans $(p=0.65)$. PET data were reconstructed via filtered backprojection (image size:128 $\times 128 \times 63$, voxel size: $2.57 \times 2.57 \times 2.43 \mathrm{~mm}$, zoom:2, filter: $4 \mathrm{~mm}$ Gaussian) and corrected for radioactive decay, scatter, random coincidences, scanner normalization, and detector deadtime.

\section{3 | MRI acquisition}

T1-weighted 3.0 T MRI scans were acquired on a SIGNA 750 (General Electric) for image coregistration and region of interest (ROI) definition. The SIGNA 750 acquisition used a 
high resolution volumetric spoiled gradient sequence (TI/TE/TR:450/3.2/8.2 ms, flip angle: $12^{\circ}$, slice thickness: $1 \mathrm{~mm}$ no gap, FOV:256, matrix size:256 $\times 256 \times 156$ ).

\section{4 | Data analysis}

PET data were realigned frame-by-frame to correct for motion (SPM12), and then HYPRLR was implemented for time-activity curve (TAC) denoising (6 mm; MATLAB2015a) (Floberg et al., 2012). The T1 MRI and FreeSurfer anatomical ROI segmentation were then coregis-tered to the PET data. Parametric DVR images were generated using MRTM2 (MATLAB2015a), which allows for the estimation of DVR given a reference region with negligible specific binding (corpus cal-losum FreeSurfer ROI; volume $=3.06 \pm 0.55 \mathrm{~cm}^{3}$ ) (Brody et al., 2006). Based on previous work with $\left[{ }^{18} \mathrm{~F}\right]$ nifene in nonhuman primates, a linearization time ( $\mathrm{t}^{*}$ ) of $10 \mathrm{~min}$ and a scan duration of 90 min was used. First, $k_{2}^{\prime}$ was estimated voxel-wise from the 3-parameter multilinear reference tissue method (MRTM), given by (Ichise et al., 2003):

$$
C(T)=R_{1} k_{2}^{\prime} \int_{0}^{T} C^{\prime}(t) d t-k_{2} \int_{0}^{T} C(t) d t+R_{1} C^{\prime}(T),
$$

where $C(T)$ is the voxel activity concentration, $k_{2}$ is the tracer clearance rate from tissue, and $R_{1}$ is the relative tracer deliver $\left(K_{1} / K_{1}^{\prime}\right)$. The prime sign indicates the parameters for the reference region. The median $k_{2}^{\prime}$ from each scan was used (coefficient of variation: $16 \%$ ) over the ROI-based estimation (19\%). Other studies have also demonstrated the same level of variability between voxel-based and ROI-based $\mathrm{k}_{2}^{\prime}$ estimation (Wu \& Carson, 2002). The $k_{2}^{\prime}$ estimates were tested for significant differences between test and retest with a related samples Wilcoxon signed rank tests (IBM SPSS Statistics 21). Then, the median $k_{2}^{\prime}$ was used as an input to MRMT2, given by (Ichise et al., 2003):

$$
C(T)=R_{1}\left(k_{2}^{\prime} \int_{0}^{T} C^{\prime}(t) d t+C^{\prime}(T)\right)-k_{2} \int_{0}^{T} C(t) d t .
$$

DVR can then be calculated as $R_{1} k_{2}^{\prime} / k_{2}$. MRTM2 DVR estimation was also performed using an a priori $k_{2}^{\prime}$ of $0.20 \mathrm{~min}^{-1}$ based on a 1-tissue compartmental model with an arterial input function performed in rhesus monkey (Hillmer, Wooten, Slesarev, et al., 2013) to evaluate the use of a population $k_{2}^{\prime}$ parameter.

The T1 MRI and FreeSurfer defined ROIs were also coregistered to the retest PET data (realigned by frame), and DVR estimation was similarly performed in PET space. Mean DVR values were extracted from parametric DVR images for the thalamus $\left(14.1 \pm 1.8 \mathrm{~cm}^{3}\right)$, stria-tum $\left(17.2 \pm 1.8 \mathrm{~cm}^{3}\right)$, cerebellum $\left(108.0 \pm 6.7 \mathrm{~cm}^{3}\right)$, hippocampus $\left(8.1 \pm 0.5 \mathrm{~cm}^{3}\right)$, anterior cingulate $\left(5.2 \pm 0.9 \mathrm{~cm}^{3}\right)$, frontal cortex $\left(78.0 \pm 9.7 \mathrm{~cm}^{3}\right)$, temporal cortex $(67.7 \pm 4.5$ $\left.\mathrm{cm}^{3}\right)$, parietal cortex $\left(54.2 \pm 7.4 \mathrm{~cm}^{3}\right)$, and rostral cortex $\left(33.9 \pm 5.8 \mathrm{~cm}^{3}\right)$. Mean DVRs were also extracted for hand-drawn lateral geniculate nucleus $\left(0.26 \pm .04 \mathrm{~cm}^{3}\right)$ and a combination (due to limited resolution) of substantia nigra and ventral tegemental area $\left(1.65 \pm 0.2 \mathrm{~cm}^{3}\right)$ ROIs. 


\section{5 | Reproducibility analysis}

Test-retest reproducibility was evaluated by calculating the test-retest variability (TRV), absolute test-retest variability (aTRV), and intraclass correlation coefficient (ICC). The TRV was calculated as the difference between the test and retest DVRs as a percentage of the average of the test and retest DVRs, according to (Hammers et al., 2007):

$$
\mathrm{TRV}=\frac{\mathrm{DVR}_{\text {test }}-\mathrm{DVR}_{\text {retest }}}{\left(\mathrm{DVR}_{\text {test }}+\mathrm{DVR}_{\text {retest }}\right) / 2} \times 100 .
$$

The aTRV was calculated as the absolute value of the TRV. The ICC, which is an estimate of the reliability of the two sets of DVR measurements within the same subject, varies from -1 (no reliability) to 1 (total reliability, i.e., identical test and retest), and commonly interpreted as poor (ICC < 0.4), fair ( $0.4<$ ICC < 0.75), or excellent (ICC > 0.75) (British Standards Institution, 1994). ICC was calculated using a one-way effects model as:

$$
I \mathrm{CC}=\frac{\mathrm{MSBS}-\mathrm{MSWS}}{\mathrm{MSBS}+(k-1) \mathrm{MSWS}}
$$

where MSBS is the mean sum of squares between subjects, MSWS is the mean sum of squares within subjects, and $k$ is the number of within subject measurements (in the testretest paradigm, $k=2$ ) (Shrout \& Fleiss, 1979). The TRV and aTRV are reported as mean \pm standard deviation, while the ICC is reported with its associated $95 \%$ confidence interval. TRV and aTRV were each tested against the null hypothesis of no variability with a 1sample Wilcoxon signed rank test (IBM SPSS Statistics 21).

\section{3 | RESULTS}

There were no adverse effects (i.e., changes in vital signs or relevant laboratory tests) that were attributable to the administration of radio-tracer in any of the eight subjects. TACs, expressed as standard uptake value across subjects, are shown in Figure 1 for representative regions. The highest uptake was observed in the thalamus, which peaked prior to $10 \mathrm{~min}$ postinjection. The lateral geniculate nucleus and the substan-tia nigra/ventral tegmental area demonstrated similar kinetics as the thalamus. Cortical and subcortical regions showed rapid uptake, peaking at approximately $5 \mathrm{~min}$. The corpus callosum showed markedly lower uptake than other regions. All regions demonstrated fast clearance. Target to corpus callosum ratios are shown in Figure 2, where ratios range from 1 to 2.5 and temporal stability occurs after $20 \mathrm{~min}$.

The estimated $k_{2}^{\prime}$ values using MRTM are summarized in Table 1 . There was no significant difference between test and retest $k_{2}^{\prime}(p=0.30)$. The $k_{2}^{\prime}$ TRV $(11 \pm 23 \%)$ was moderately positive, but was not significantly different from zero $(p=0.24)$. An a priori $k_{2}^{\prime}$ (as opposed to a test $k_{2}^{\prime}$ and a retest $k_{2}^{\prime}$ ) was investigated to determine its influence on the DVR test-retest variability. The percent difference in the estimated $k_{2}^{\prime}$ parameters from the a priori $k_{2}^{\prime}$ was 29 $\pm 12 \%$. Voxel-wise comparison between the parametric DVR images generated from the 
estimated $k_{2}^{\prime}$ and the a priori $k_{2}^{\prime}$ revealed no significant differences using a paired $t$ test in SPM12 $(p<.001,>50$ voxels). Additionally, the test-retest variability and ICCs were not significantly different (paired $t$ test, $p<.05$ ), supporting the use of a single population $k_{2}^{\prime}$ parameter. Figure 3 shows average test and retest parametric DVR images. $\left[{ }^{18} \mathrm{~F}\right]$ Nifene DVR was highest in the thalamus, moderate in the striatum, hippocampus, anterior cingulate, and cerebellum, and lowest in neocortical regions.

The test-retest DVR reproducibility metrics are summarized in Table 2 . The magnitude of the deviation between test and retest (aTRV) was significantly different from zero in all ROIs. However, the TRV was not significantly different from zero in any ROI. The ICC was high in the cerebellum, temporal and parietal cortices, and striatum, moderate in the frontal and rostral cortices, hippocampus, anterior cin-gulate, and low in the thalamus.

Temporal stability of DVR estimates was investigated by truncating dynamic data at $10 \mathrm{~min}$ intervals and comparing to the full 90 min derived estimates. Figure 4 demonstrates minimal change in DVR estimation when scan duration is truncated. The percent difference for 30 , $40,50,60,70$, and $80 \mathrm{~min}$ datasets compared to the full $90 \mathrm{~min}$ dataset is $5.6 \pm 3.2 \%, 3.6$ $\pm 2.6 \%, 2.4 \pm 1.7 \%, 1.6 \pm 1.1 \%, 1.0 \pm 0.6 \%$, and $0.4 \pm 0.3 \%$, respectively, for the highest binding region (thalamus). For comparison, a low binding region (frontal cortex) has respective percent differences of $4.9 \pm 2.0 \%, 2.8 \pm 1.6 \%, 1.5 \pm 1.3 \%, 0.9 \pm 1.0 \%, 0.5 \pm 0.7 \%$, $0.2 \pm 0.5 \%$. Dataset truncation did not significantly affect the ICCs $(p>.05)$.

\section{4 | DISCUSSION}

This study reports the first-in-human evaluation of $\left[{ }^{18} \mathrm{~F}\right]$ nifene indicating no adverse effects and reflecting the known distribution of $\alpha 4 \beta 2 * n A C h R s$ in the healthy brain consistently demonstrated in imaging studies (Brody et al., 2006; Kimes et al., 2003). MRTM2 DVR estimation demonstrated test-retest variability comparable to most PET radioligands, with a magnitude ranging from 6 to $11 \%$ with no systematic difference between test and retest, for these studies with injected doses of $200 \pm 3.7 \mathrm{MBq}$. Whole-body dosimetry studies are ongoing and will likely yield a higher allowable injected dose and improved imaging statistics.

The region of highest $\left[{ }^{18} \mathrm{~F}\right]$ nifene DVR was the thalamus, which was also the region of lowest ICC. The ICC uses information about the systematic difference between methods within a subject (i.e., test and retest) and information about the random measurement variation between subjects. The magnitude of the DVR value does not influence the value of the ICC. The ICC equation shows that for a given within-subject variability a higher between-subject variability will lead to a higher ICC. For instance, the hippocampus and the temporal cortex have a similar within-subject variabilityof $7 \%$, but the hippocampus has a between-subject variability of $9 \%$ and the temporal cortex has a between-subject variability of $12 \%$, which lead to ICCs of 0.66 and 0.83 , respectively. However, in the thalamus, the within-subject variability is $11 \%$ and the between-subject variability is $9 \%$, leading to a low ICC of 0.26 in this small sample size. 
Scan duration is an important factor in the translation of a novel radiotracer for use in human research. The scan needs to be long enough to obtain stable estimate of parameters related to receptor density as well as to provide sufficient counting statistics, however, it is also desirable to shorten the scan duration to minimize the potential discomfort for subjects lying motionless for an extended period of time. For imaging the a $4 \beta 2 * n A C h R s$, the commonly used $\left[{ }^{18} \mathrm{~F}\right] 2 \mathrm{FA}$ requires $6-8 \mathrm{hr}$ of scan time whereas the currently used $\left[{ }^{18} \mathrm{~F}\right]$ nifene requires a $40 \mathrm{~min}$ acquisition (percent difference compared to $90 \mathrm{~min}$ of data $<4 \%$ ). This is faster than the time required by high affinity a $4 \beta 2 * \mathrm{nAChR}$ radiotracers such as $\left[{ }^{18} \mathrm{~F}\right] \mathrm{AZAN}$, $\left[{ }^{18} \mathrm{~F}\right] \mathrm{XTRA}$, or $\left[{ }^{18} \mathrm{~F}\right]$ flubatine. Furthermore, truncating the scan time had no effect on the test-retest ICC.

There are several limitations to the current study. There was no arterial blood sampling in this initial investigation of the in vivo time course of $\left[{ }^{18} \mathrm{~F}\right]$ nifene in the human brain. Our previous $\left[{ }^{18} \mathrm{~F}\right]$ nifene studies in rhesus monkeys showed that MRTM2 estimations were similar to those from a 1-tissue kinetic analysis when using an arterial input function (Hillmer, Wooten, Slesarev, et al., 2013). The corpus callosum was selected as a reference region based on previous human imaging studies of a $4 \beta 2 *$ nAChRs (Brody et al., 2006), which reported displaceable binding in the cerebellum but not in the corpus callosum following cigarette smoking. The corpus callosum has also been used as a reference region in human a $4 \beta 2 *$ PET studies of Parkinson's disease (Meyer et al., 2009). It should be noted that white matter regions may differ from gray matter regions in the nondisplacable distribution volume, however these regions are typically devoid of nAChRs. Future studies with arterial sampling may be needed to carefully examine the validity of corpus callosum as reference region to characterize potential parameter bias for the quantification of $a 4 \beta 2 *$ nAChR binding with $\left[{ }^{18} \mathrm{~F}\right]$ nifene. In this first-in-human study, there was no effort made to detect the effect of smoking status on $\left[{ }^{18} \mathrm{~F}\right]$ nifene binding. Detailed measures of smoking status such as carbon monoxide, nicotine, or cotinine are needed to assess their effects on $\left[{ }^{18} \mathrm{~F}\right]$ nifene binding. Lastly, recent studies have reported on cellular trafficking of nAChR (reviewed in St John, 2009). Since $\left[{ }^{18} \mathrm{~F}\right]$ nifene exhibited relatively fast kinetics which is partially attributed to the lower basicity of the 3-pyrrolinyl ring, our expectation is that it may not be sequestered in intracellular vesicles. However, further studies are required to confirm if any $\left[{ }^{18} \mathrm{~F}\right]$ nifene is being vescicularized during the course of a PET scan.

\section{5 | CONCLUSION}

The first-in-human findings suggest that $\left[{ }^{18} \mathrm{~F}\right]$ nifene is a reliable and reproducible radiotracer for $a 4 \beta 2 *$ nAChRs when estimating DVR with MRTM2. The rapid kinetics allow for scan times as short as $40 \mathrm{~min}$, which is beneficial for studies in compromised populations that cannot tolerate long scans.

\section{Acknowledgments}

Funding information

This research was funded by the National Institute of Health (R01 AG029479) and the National Institute of Child Health and Human Development (U54 HD090356). 
The authors would like to thank the UW-Madison cyclotron group, project managers, and technologist who provided invaluable help in producing radioisotopes, and coordinating and conducting PET scans, respectively. This research was funded by the National Institute of Health (R01 AG029479) and the National Institute of Child Health and Human Development (U54 HD090356).

\section{References}

British Standards Institution. Accuracy (trueness and precision) of measurement methods and results: Basic methods for the determination of repeatability and reproducibility of a standard measurement method. London: HMO; 1994. BS ISO 5725 part 2

Brody AL, Mandelkern MA, London ED, Olmstead RE, Farahi J, Scheibal D, ... Mukhin AG. Cigarette smoking saturates brain a $4 \beta 2$ nicotinic acetylcholine receptors. Archives of General Psychiatry. 2006; 63:907-915. [PubMed: 16894067]

Constantinescu CC, Garcia A, Mirbolooki MR, Pan ML, Mukherjee J. Evaluation of $\left[{ }^{18} \mathrm{~F}\right]$ Nifene biodistribution and dosimetry based on whole-body PET imaging of mice. Nuclear Medicine and Biology. 2013; 40(2):289-294. [PubMed: 23265670]

Floberg JM, Mistretta CA, Weichert JP, Hall LT, Holden JE, Christian BT. Improved kinetic analysis of dynamic PET data with optimized HYPR-LR. Medical Physics. 2012; 39(6):3319-3331. [PubMed: 22755714]

Gotti C, Clementi F. Neuronal nicotinic receptors: From structure to pathology. Progress in Neurobiology. 2004; 74(6):363-396. [PubMed: 15649582]

Hammers A, Asselin MC, Turkheimer FE, Hinz R, Osman S, Hot-ton G, ... Koepp MJ. Balancing bias, reliability, noise properties and the need for parametric maps in quantitative ligand PET:

$\left[{ }^{11} \mathrm{C}\right.$ ]diprenorphine test-retest data. NeuroImage. 2007; 38:82-94. [PubMed: 17764977]

Hillmer AT, Tudorascu DL, Wooten DW, Lao PJ, Barnhart TE, Ahler EO, ... Christian BT. Changes in the a $4 \beta 2 *$ nicotinic acetylcholine system during chronic controlled alcohol exposure in nonhuman primates. Drug Alcohol Depend. 2014; 138:216-219. [PubMed: 24602361]

Hillmer AT, Wooten DW, Farhoud M, Barnhart TE, Mukherjee J, Christian BT. The effects of lobeline on $a 4 \beta 2 *$ nicotinic acetylcholine receptor binding and uptake of $\left[{ }^{18} \mathrm{~F}\right]$ nifene in rats. Journal of Neuroscience Methods. 2013; 214(2):163-169. [PubMed: 23370310]

Hillmer AT, Wooten DW, Moirano J, Slesarev M, Barnhart TE, Engle JW, ... Christian BT. Specific a $4 \beta 2$ nicotinic acety-lecholine receptor binding of [F-18]nifene in the rhesus monkey. Synapse. 2011; 65(12):1309-1318. [PubMed: 21674627]

Hillmer AT, Wooten DW, Slesarev MS, Ahlers EO, Barnhart TE, Murali D, ... Christian BT. PET imaging of $a 4 \beta 2 *$ nicotinic acetylcholine receptors: Quantitative analysis of ${ }^{18} \mathrm{~F}$-nifene kinetics in the nonhuman primate. Journal of Nuclear Medicine. 2012; 53:1471-1480. [PubMed: 22851633]

Hillmer AT, Wooten DW, Slesarev MS, Ahlers EO, Barnhart TE, Schneider ML, ... Christian BT. Measuring a $4 \beta 2 *$ nicotinic acetylcholine receptor density in vivo with $\left[{ }^{18} \mathrm{~F}\right]$ nifene PET in the nonhuman primate. Journal of Cerebral Blood Flow \& Metabolism. 2013; 33:1806-1814. [PubMed: 23942367]

Ichise M, Liow JS, Lu JQ, Takano A, Model K, Toyama H, ... Carson RE. Linearized reference tissue parametric imaging methods: Application to $\left[{ }^{11} \mathrm{C}\right] \mathrm{DASB}$ positron emission tomography studies of the serotonin transporter in human brain. Journal of Cerebral Blood Flow \& Metabolism. 2003; 23:1096-1112. [PubMed: 12973026]

Kimes AS, Chefer SI, Matochik JA, Contoreggi CS, Vaupel DB, Stein EA, Mukhin AG. Quantification of nicotinic acetylcholine receptors in the human brain with PET: Bolus plus infusion administration of 2-[ ${ }^{18}$ F]F-A85380. NeuroImage. 2008; 39:717-727. [PubMed: 17962044]

Kimes AS, Horti AG, London ED, Chefer SI, Contoreggi C, Ernst M, ... Mukhin AG. 2-[18F] FA-85380: PET imaging of brain nicotinic acetylcholine receptors and whole body distribution in humans. The FASEB Journal. 2003; 17(10):1331-1333. [PubMed: 12759330]

Kuwabara H, Gao Y, Stabin M, Coughlin J, Nimmagadda S, Dannals RF, ... Horti AG. Imaging a4 $\beta 2$ Nicotinic Acetylcholine Receptors (nAChRs) in baboons with $\left[{ }^{18} \mathrm{~F}\right]$ XTRA, a radioligand with improved specific binding in extra-thalamic regions. Molecular Imaging and Biology. 2016; 19(2): 280-288. 
Liang C, Patel IH, Pan ML, Mukherjee J. Affinity and selectivity of nifene for human nicotinic receptor subtypes. Journal of Nuclear Medicine. 2016; 57(6suppl):1798. [PubMed: 27609786]

Lindstrom J, Anand R, Peng X, Gerzanich V, Wang F, Li Y. Neuronal nicotinic receptor subtypes. Annals of the New York Academy of Sciences. 1995; 757:100-116. [PubMed: 7611667]

Meyer PM, Strecker K, Kendziorra K, Becker G, Hesse S, Woelpl D, ... Schwarz J. Reduced a4ß2*_ nicotinic acetylcholine receptor binding and its relationship to mild cognitive and depressive symptoms in par-kinson disease. Archives of General Psychiatry. 2009; 66(8):866-877. [PubMed: 19652126]

Nybäck H, Halldin C, Åhlin A, Curvall M, Eriksson L. PET studies of the uptake of (S)- and (R)$\left[{ }^{11} \mathrm{C}\right]$ nicotine in the human brain: Difficulties in visualizing specific receptor binding in vivo. Journal of Psychopharmacology. 1994; 115:31.

Pichika R, Easwaramoorthy B, Collins D, Mukherjee J. Nicotinic a $4 \beta 2$ receptor imaging agents: Part II. Synthesis and biological evaluation of 2-[ $\left.{ }^{18} \mathrm{~F}\right]$ fluoro-3-[2-((S)-3-pyrrolinyl)methoxy]pyridine $\left({ }^{18} \mathrm{~F}-\right.$ nifene $)$ in rodents and imaging by PET in nonhuman primate. Nuclear Medicine and Biology. 2006; 33(3):295-304. [PubMed: 16631077]

Shrout PE, Fleiss JL. Intraclass correlations: Uses in assessing rater reliability. Psychological Bulletin. 1979; 86(2):420. [PubMed: 18839484]

St John PA. Cellular trafficking of nicotinic acetylcholine receptors. Acta Pharmacologica Sinica. 2009; 30(6):656. [PubMed: 19498414]

$\mathrm{Wu}$ Y, Carson RE. Noise reduction in the simplified reference tissue model for neuroreceptor functional imaging. Journal of Cerebral Blood Flow \& Metabolism. 2002; 22:1440-1452. [PubMed: 12468889] 
Time Activity Curves for $\left[{ }^{18} \mathrm{~F}\right]$ Nifene

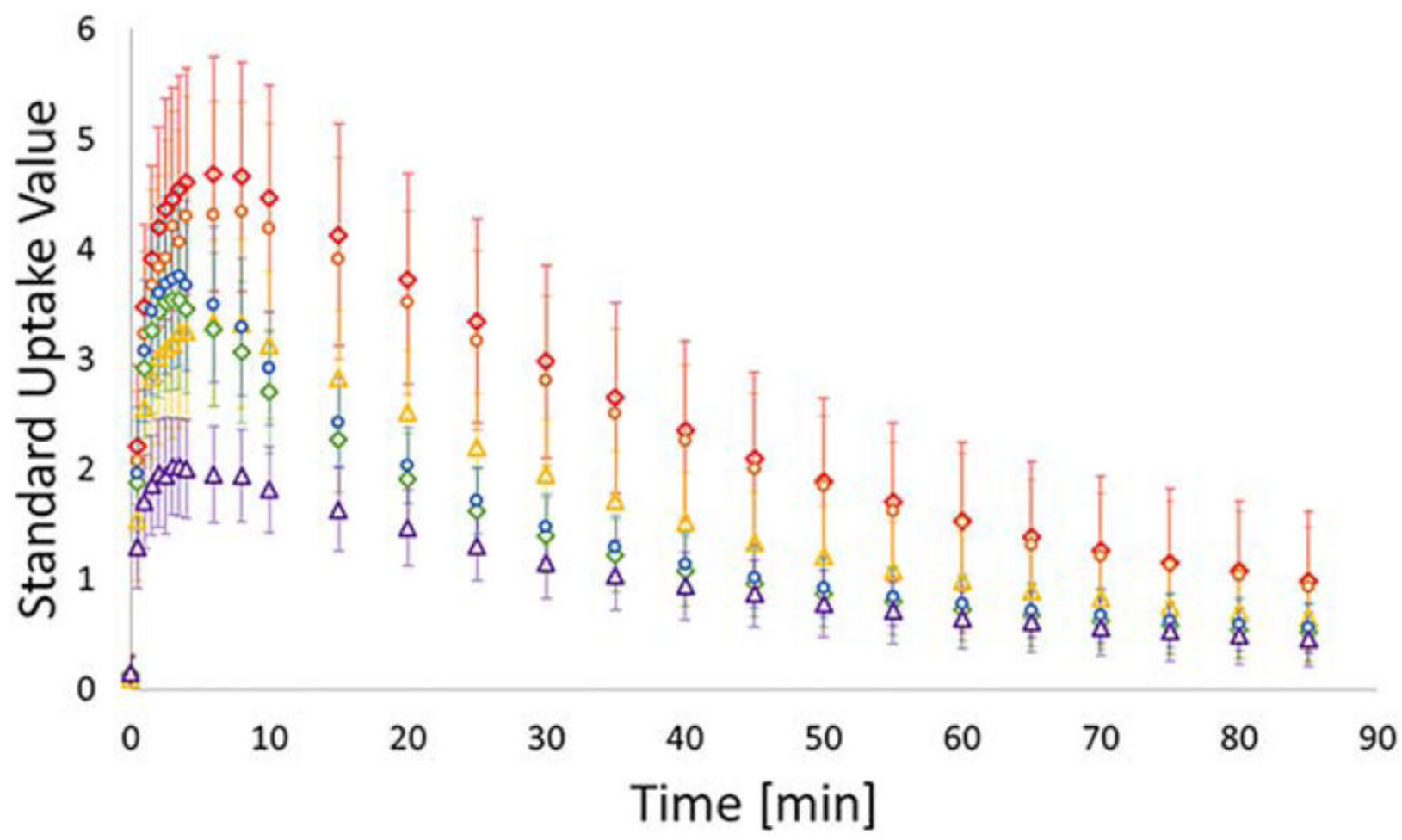

$\diamond$ Thalamus

- Lateral Geniculate Nucleus

$\Delta$ Susbtantia Nigra/Ventral Tegemental Area $\diamond$ Frontal cortex

$\times$ Cerebellum

$\times$ Corpus Callosum

FIGURE 1.

Time activity curves (TACs) in representative regions averaged across all subjects (eight subjects, 15 scans), with standard deviation bars 
Target to Corpus Callosum Ratios for [ $\left.{ }^{18} \mathrm{~F}\right]$ Nifene

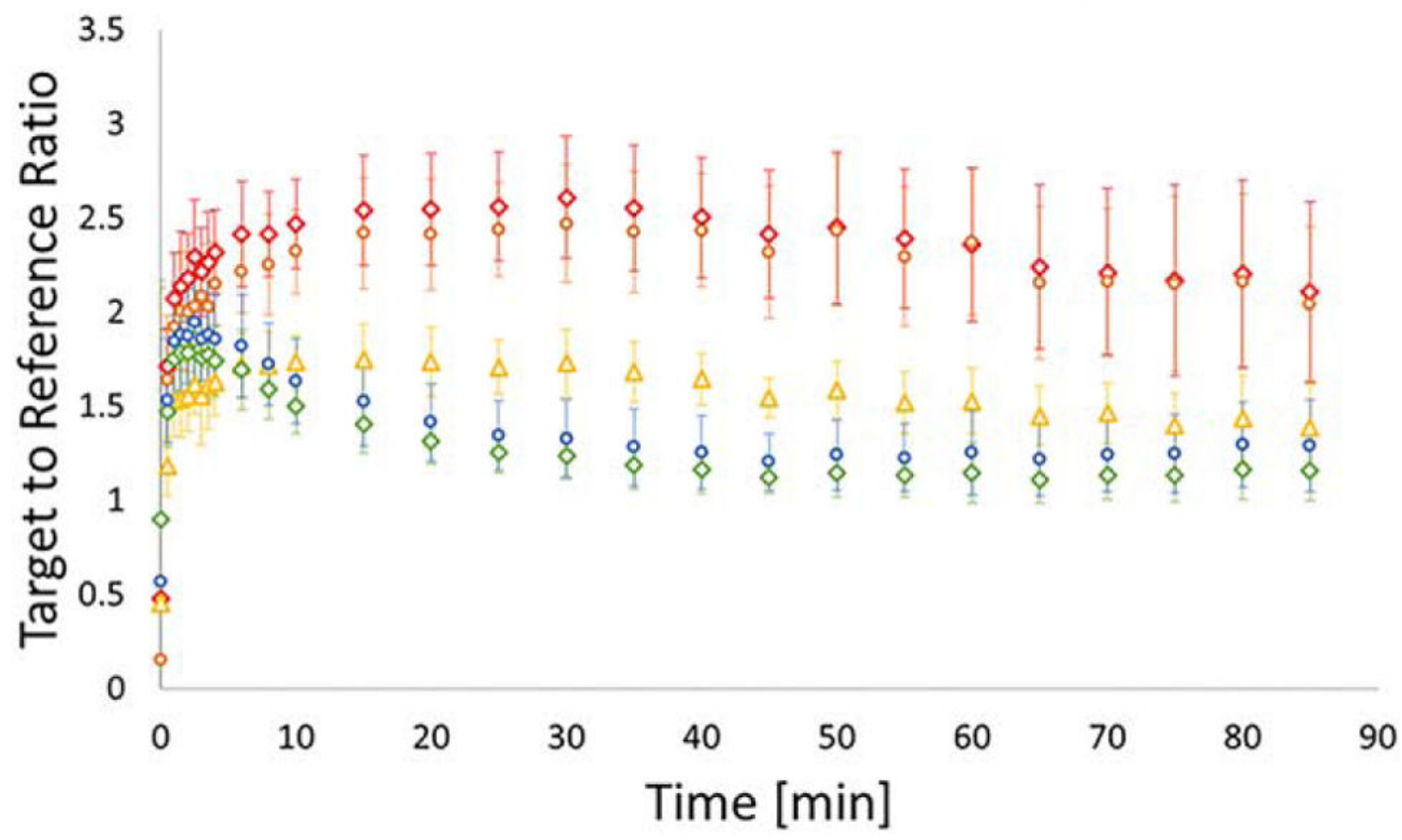

$\diamond$ Thalamus

- Lateral Geniculate Nucleus

$\triangle$ Susbtantia Nigra/Ventral Tegemental Area $\diamond$ Frontal cortex $\times$ Cerebellum

FIGURE 2.

Target to reference region ratio curves in representative regions averaged across all subjects (eight subjects, 15 scans), with standard deviation bars. The highest binding region (thalamus) plateaus within 20 min postinjection, indicating fast kinetics 


\section{Parametric [ $\left.{ }^{18} \mathrm{~F}\right]$ nifene DVR images}

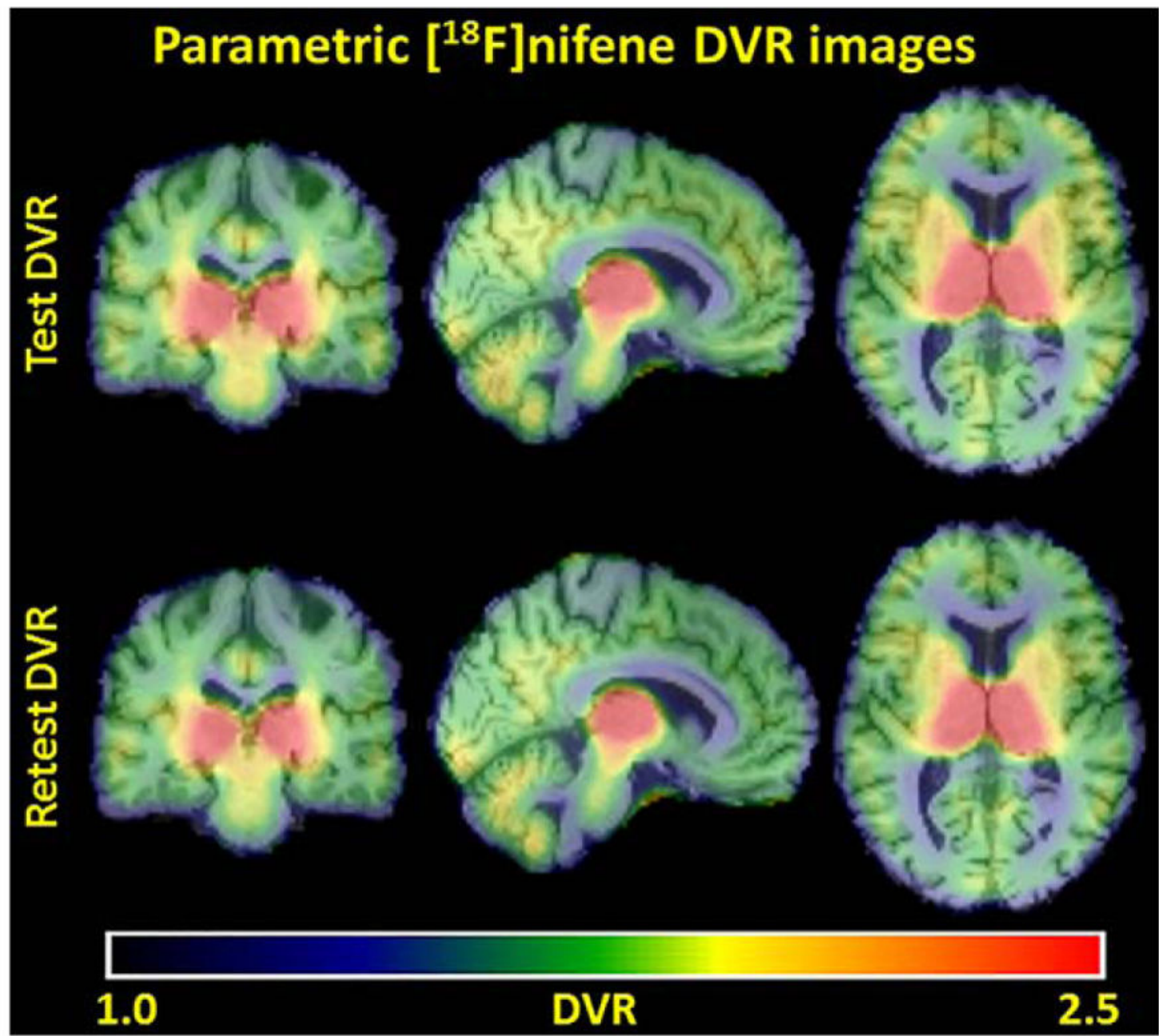

FIGURE 3.

Parametric DVR images showing the distribution of $\left[{ }^{18} \mathrm{~F}\right]$ nifene in brain and the average test and retest scans for seven healthy subjects 


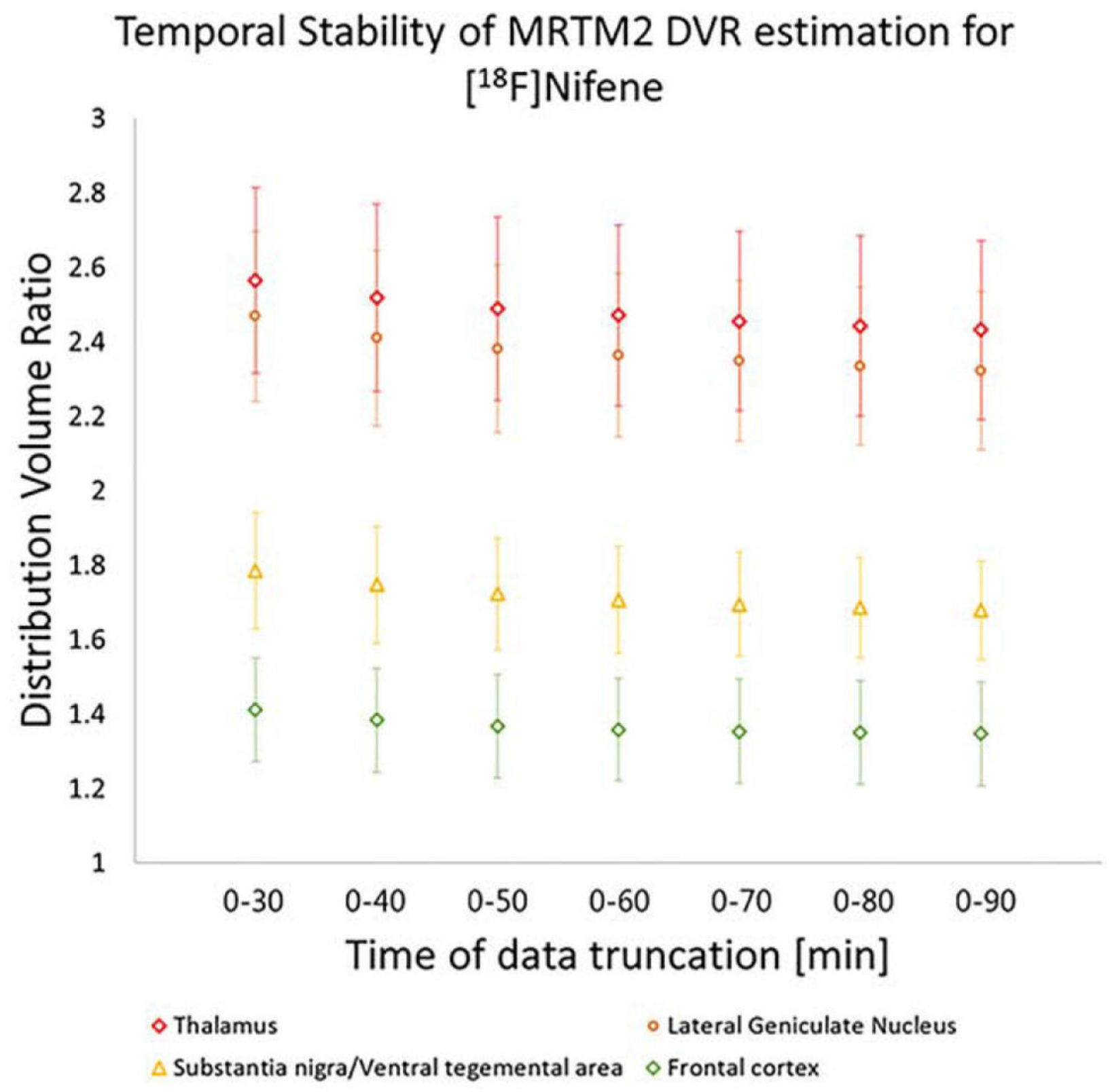

FIGURE 4.

DVR and standard deviation bars from truncated datasets for $\left[{ }^{18} \mathrm{~F}\right]$ nifene using MRTM2 

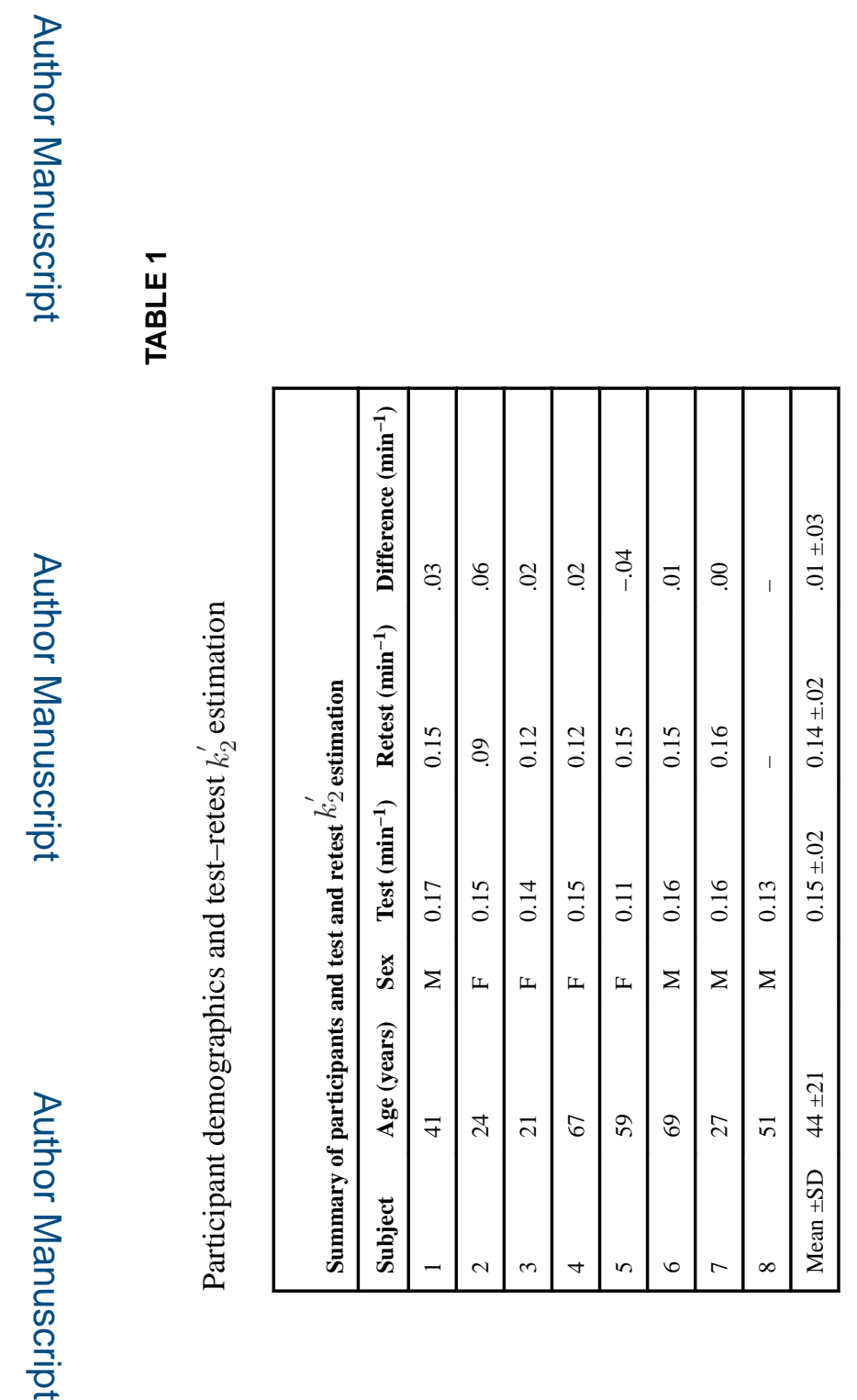


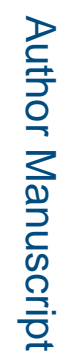
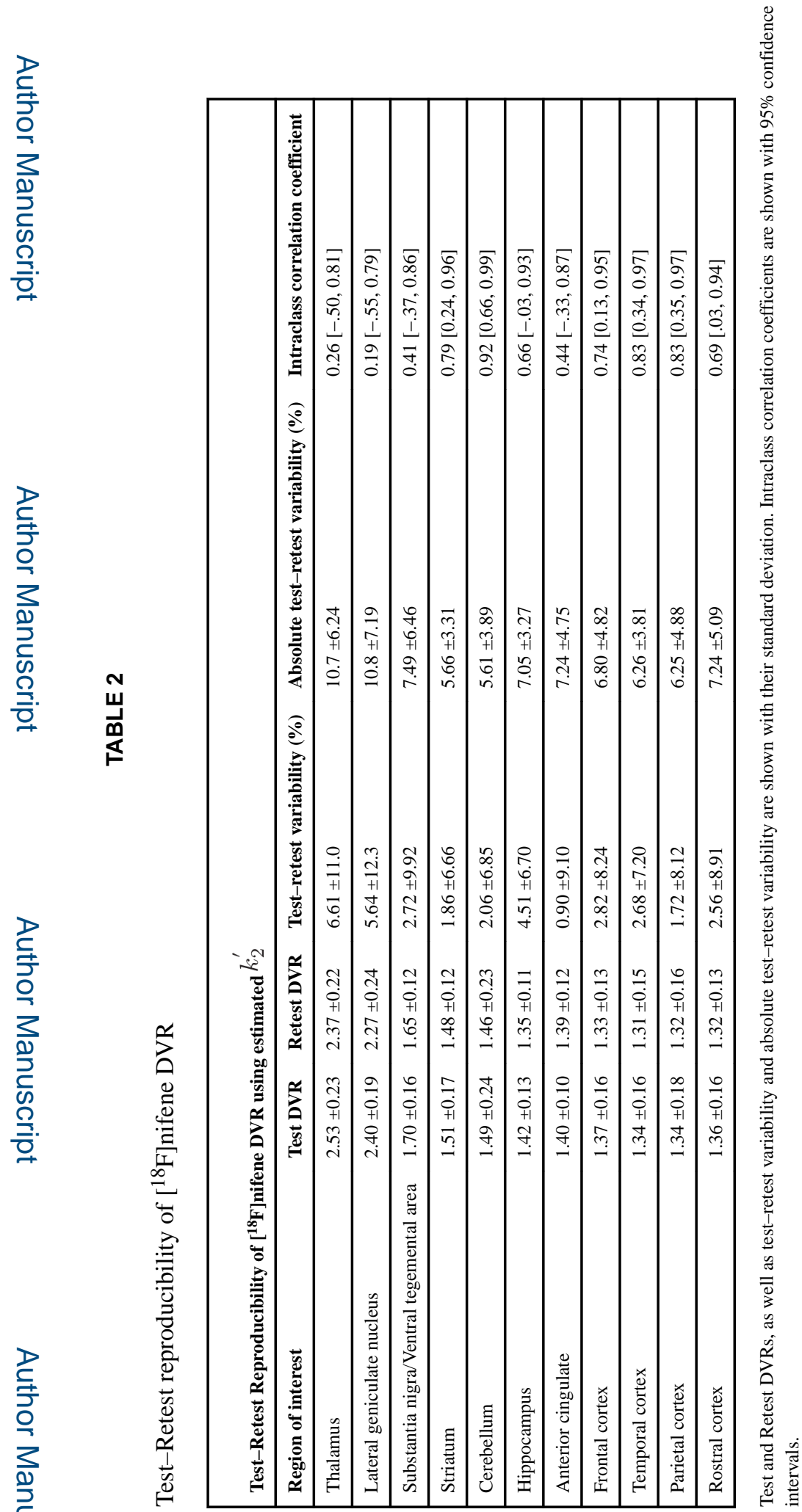

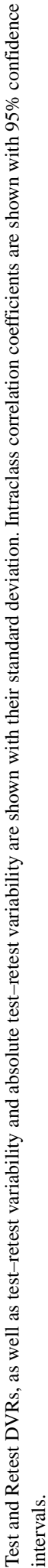

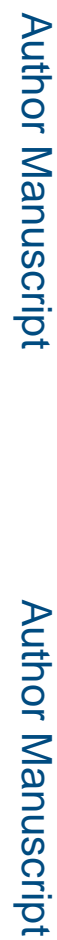

Synapse. Author manuscript; available in PMC 2018 August 01. 